\title{
The Impact of COVID-19 on Breast Surgery Fellowships
}

\author{
Ann-Kristin U. Friedrich ${ }^{1}$ Joseph A. DiComo² $\cdot$ Mehra Golshan $^{1}$
}

Accepted: 7 September 2021 / Published online: 22 October 2021

(c) The Author(s), under exclusive licence to Springer Science+Business Media, LLC, part of Springer Nature 2021

\begin{abstract}
Purpose of Review Across the world, medical training has been affected by the COVID-19 virus and this has changed the way physicians are educated. What is less clear is the effect of the pandemic on breast surgical oncology fellows who were in training during this time. This review discusses the experience of breast surgical oncology fellows during the pandemic and how fellowships adapted to preserve the educational experience and conserve the quality of training.

Recent Findings The challenges and changes experienced by breast surgery fellows during the COVID-19 pandemic have proved to be sudden shifts in not only fellowship training, but future patient care and research opportunities, all while confronting the global impact of a deadly pandemic. While experiences between fellowships varied, the similarities and differences encountered highlight the regional and temporal differences in how fellowships responded to the pandemic.

Summary Breast surgical oncology fellowship is one year long, with every day allocated to ensure the surgeon has a deep understanding of the multidisciplinary approach to this ever-evolving field. As the pandemic spread and affected different regions with varying severity, elective cases were canceled, resources were re-allocated, and uncertainty abounded. At the same time, novel approaches to fellowship training were rapidly implemented. It will take time and additional research to fully understand the long-term consequences for trainees affected during their breast surgery fellowship.
\end{abstract}

Keywords Breast surgery fellowship $\cdot$ Breast surgical oncology $\cdot$ COVID-19 $\cdot$ Postgraduate education

\section{Introduction}

The coronavirus Sars-Cov-2 pandemic (COVID-19) has had an unprecedented impact on modern society [1]. The extent of this impact was initially unimaginable when the novel coronavirus disease was first brought to the attention of the medical community and initial reports underestimated its pathogenicity and transmissibility [2]. A consequence of the pandemic was haste changes in medical practice in an attempt to contain the rapidly spreading virus [3•]. During its first peak, these practice changes have led to a near total

This article is part of the Topical Collection on Breast Cancer Management During the COVID-19 Pandemic

Ann-Kristin U. Friedrich

ann.friedrich@yale.edu

1 Department of Surgery, Yale University School of Medicine, 310 Cedar Street, New Haven, CT 06510, USA

2 Department of Surgery, Women and Infant's, Providence, RI, USA stop of elective procedures in affected states. This had specific consequences for physician training and education. This is especially true for fellowships in predominantly elective specialties such as breast surgery, which typically only lasts one year.

Usually, postgraduate training for physicians takes multiple years; most residency programs require between 3 and 5 years to complete. This is frequently extended due to both mandatory and elective research endeavors. Fellowship training is usually completed after residency training and can vary in length depending on the subspecialty. This is somewhat different for breast surgery fellows: the fellowship lasts one year, and only a portion of it is dedicated exclusively to breast surgery. Its interdisciplinary aspect requires the breast fellow to spend time outside the operating room and rotate through numerous subspecialties, among these radiology, medical oncology, plastic surgery, pathology, and radiation oncology. A certain minimum number of specialty-specific breast surgery operations have to also be completed during the breast surgical portion in order to successfully complete the fellowship [4]. Fellows have one year to meet these 
requirements, but what happens in the event of a worldwide pandemic that suddenly interrupts that year?

\section{Consequences on Breast Fellow Training}

The pandemic has affected breast surgery fellowships in numerous ways, and it is fair to say that we have both lost and gained training opportunities. Changes to regular training resulted from the way we changed clinical practice and involved number of performed operative cases, didactic sessions, research projects, conferences, patient care, and time off work due to disease or quarantine requirements in addition to numerous other learning opportunities which are difficult to quantitate.

\section{Practice Changes}

The unprecedented challenges to oncologic care during the pandemic included developing a flexible approach to patient care. This involved changes in treatment timing, allocation of limited resources, limiting transmissibility through infection reduction measures, and implementing increased safety precautions for physicians, healthcare staff, and patients [5••]. In order to minimize patient exposure to the virus and to preserve healthcare resources during the initial peak, hospitals nationwide were asked to significantly decrease the number of elective and non-urgent procedures, including those for cancer. A wide variety of diagnostic and therapeutic cancer management strategies with a wide variation in the timing and role of surgery were suggested and implemented [6]. This further complicated and pushed the boundaries of agreed and accepted recommendations for safe cancer management. In an attempt to help with the prioritization of operations, the COVID-19 Pandemic Breast Cancer Consortium published specific recommendations about operative decision-making for benign and malignant breast diseases $[7 \bullet \bullet]$. The extent to which these recommendations were practiced was however left to discretion of the individual hospital. As the virus affected some regions of the USA more severely with varying levels of subsequent responses, it consequently had varying effects on breast surgery fellowships [8].

New York and New England were among the first areas to be affected severely [9]. During that time, hospitals ceased elective patient care, applied disaster management strategies to care for critically sick patients, and restructured and repurposed teams, resources, and equipment. For breast cancer patients treated according the published recommendations by the Pandemic Breast Cancer Consortium, there was an increase in the use of neoadjuvant chemotherapy and endocrine therapy, as well as delayed breast cancer screening and imaging. This in turn led to less breast operative procedures and less cancer detection through screening [10•]. At this time, it is difficult to understand and might still be too early to analyze the oncologic impacts of this delayed surgical approach. However, retrospective data analysis shows reassuring results that might be extrapolated to at least those patients with DCIS [11]. At this time, it is well understood that a significant delay in operative management for invasive cancer negatively affects patient survival [12]; what constitutes a significant delay, however, is controversial. As the duration of the pandemic has exceeded initial estimates, which have proved uncertain and unreliable, it consequently has increased the number of clinicians uncomfortable with increasing delays before surgical oncologic treatment. Another factor which must be considered is the fact that unlike many other surgical subspecialties, breast surgery represents a high operative volume with a low risk for significant utilization of healthcare resources and hospital capacity [13]. This may imply that most breast surgery fellows currently in training may be able to continue their involvement in operative cases, in the event of a further prolonged COVID-19 pandemic.

\section{Operative Cases}

At the authors' institutions in New England, almost all elective cases were essentially canceled or delayed and rescheduled. This significantly decreased our operative experiences during the months of March, April and May 2020. Not all breast oncologic procedures appeared to be affected similarly; our own case volumes during and after these months demonstrated an increase in lumpectomies and a decrease in mastectomies. This was most evident in the rate of prophylactic mastectomies. In addition, we experienced a decrease in immediate reconstruction after breast surgery, with the greatest reduction in cases utilizing autologous tissue. However, in our particular instances, this did not significantly affect our ability to meet the minimum number of cases needed to graduate as we were fortunate to have been at high-volume centers and had met most minimum operative requirements well before the pandemic induced practice changes took effect. Similar experiences were noted by breast surgery fellows of other institutions with high volumes as well [8]. The decreased operative exposure is generally comparable to those of other surgical specialties, which similarly demonstrated overall reduced caseloads and logged procedure numbers for trainees [8, 14]. Fortunately for our institutions, the last months of fellowship demonstrated decreasing numbers of active COVID-19 inpatients in New England and operative volume increased as services were re-introduced. The backlog of delayed cases allowed for baseline or above baseline operative numbers in the 
months of June and July 2020. However, we assume that as COVID-19 cases were simultaneously rising in other parts of the country, other fellowships may have been affected in a similar fashion to the way our institutions were at the start of the pandemic.

\section{Didactic Sessions}

A unique consequence of social distancing for breast surgery fellows was difficulty in spending time with subspecialty mentors, primarily in the multidisciplinary fields involved in breast disease. This was particularly evident when the tight quarters of a radiology reading room or shared microscope in the pathology department were no longer an option. If fellows were fortunate enough to have these rotations before the pandemic, then they would likely have lost another opportunity to learn from other mentors on the plastic surgery, medical oncology, radiation oncology service, or any other service or learning experience that was unfortunately coincided with the pandemic affecting their region at that particular time.

To complicate matters further, many of these rotations may be at other facilities or private practices with different rules than the fellow's home institution which may have prevented them from learning from physicians who may have had profound impact on the education of the fellow. In response to this, physician leaders demonstrated their commitment to education by making novel and creative efforts to use digital and virtual resources [14]; to include breast surgery fellows in training usually intended for residents of other subspecialties; and to even provide private lectures. Didactic training occurred through virtual interfaces and at the end of Telehealth patient encounters. These innovative teaching methods are crucial to surgical education during this pandemic [15]. A consequence of this effective means of communication and learning has initiated discussions of completely virtual surgical rotations to enhance medical student education [16]; and while as a supplemental source it may be useful, we do not consider it appropriate or sufficient for fellowship training.

\section{Research and Research Forums}

COVID-19 has delayed, disrupted, or stopped basic science and clinical research across all disciplines around the world [17]. Additionally, medical and scientific conferences around the world have been either canceled or changed to a virtual format. For our generation of breast surgery fellows, the ability to present original research at national and international annual meetings has been limited to a virtual only format [18]. While virtual meetings can provide an effective medium to communicate knowledge and innovation, they lack the personal and often complex human social interactions that can lead to collaboration, mentorship, and friendship while at the same time limiting networking opportunities and access to simulation labs and hands on skills courses. Virtual meetings have also demonstrated that they are forced to condense and prioritize research to be presented, leading to missed opportunities for fellows who would have had a submission accepted in the usual in person meetings.

Enrolment in clinical trials have been significantly impacted by the pandemic $[19,20]$, with the highest decrease noted in April 2020 at 70\% across 1500 sites monitored by Medidata, a healthcare data services company. This appears to be improving, with June 2020 reporting a 38\% decrease from prepandemic levels [19]. The pandemic's impact on clinical trials can vary greatly and depends on both geography and therapeutic area, with oncology appearing to be the therapeutic area impacted least [21]. COVID-19's impact on rare diseases has spurred the NIH to conduct a survey to examine its impact on patients with rare diseases [22].

The global pandemic forced scientific research labs across the country to shut down, putting lifesaving treatment developments on hold and severely limiting the opportunities of fellows interested in pursuing research. The effect on earlycareer physicians and scientists interested in basic science research appears to be particularly at risk [23].

\section{Patient Care}

Social distancing rules have led to minimal physical contact with other physicians and patients, as well as an increased use of telemedicine [3•]. This has been adapted successfully in a wide variety of medical settings with adequate satisfaction by both patients and providers [24]. Often, teams were created to minimize exposures to other teams and their members $[5 \bullet \bullet$. The resulting implications for breast surgery trainees are less opportunities to perform physical exams and increased difficulty to contribute to multidisciplinary meetings. However, the pandemic also represented a unique opportunity to refine and develop a range of professional and empathic communication techniques relevant to the telehealth setting [25].

This transition to Telehealth and prioritization also has its limitations and downsides. Patients needing care are often afraid to seek it due to the pandemic [26]. They may not feel as if their issues or concerns are as important in the setting of a pandemic and intentionally risking their own health out of fear of unintentionally utilizing healthcare resources that may be more needed elsewhere. Interactions via telehealth may miss the visual cues we have used for the entirety of medical practice to signal a patient might be overwhelmed, confused, or emotionally 
compromised. Cancer is an intimate and emotional diagnosis; and the human interaction of personally meeting with a physician is a vital component that may not be able to be mitigated or compromised. In some cases, the fear of worsening or continued pandemic may have rushed patients to make surgical decisions they would have otherwise spent more time discussing other treatment options or mentally preparing for treatment.

\section{Direct Effects of the Virus}

The implications of the virus have also directly affected fellows and patients alike. Contracting a COVID-19 infection can be challenging for the breast surgery trainee - not only do the disease, recovery, and quarantine requirements take away time from education, they also place an additional burden on breast surgery fellows who care for their own family members during quarantine [27]. Like the rest of the population, our fellow colleagues who contracted the disease themselves had to isolate and recover and were often taken care of by colleagues of their institutions.

Of course, breast cancer patients may also get the virus, which can have devastating effects especially among the immunocompromised [28]. Accurate diagnosis of COVID19 combined with potential unknown interactions between the virus and cancer therapy has presented new and unique challenges [29]. Moreover, the high prevalence of the virus during the early stage of the pandemic has complicated diagnosis and management of numerous common breast surgical and oncologic problems. Oncologic patients are more prone to present with fever or cough, which contributes to the difficulty of an accurate COVID-19 diagnosis [30], and conversely, the concern for coronavirus may complicate the diagnosis of oncologic complications and other infectious conditions. These problems are compounded by the emphasis on telehealth and the challenges of remote diagnosis. From personal experience, it can be a challenge to remotely manage calls from patients, who are worried they might have mastitis, abscesses, or wound infections, but may instead have contracted COVID-19. Under normal circumstances, a physical exam would have easily and quickly provided clarity for these cases; however, deciding whom to see in person, whom to send to the emergency room (especially given concerns for virus exposure), and whom to evaluate remotely became a repeated and seemingly endless challenge of the pandemic. Balancing the need for a physical exam with the risk of potential exposure was a unique and ongoing challenge to many fellows in epicenters of COVID-19.

\section{Additional Responsibilities for Breast Surgery Fellows}

In our experience, the forced redeployment of breast surgery fellows to other services has been the rare exception. One of the authors (AF) has volunteered to be included in her institution's critical care backup provider pool but was ultimately not utilized in this function. She also contributed to the institution's "COVID-19 wellness message a colleague program of support," where weekly communication between randomly assigned trainees was encouraged to mitigate feelings of isolation, provide mental and emotional support, and facilitate dialogue among trainees in similar situations. Stress that is seen during surgical training at baseline [31] is manifold and was found to be heightened among surgical trainees during the pandemic [32]. These institutional programs were implemented in the hopes of improving trainees' mental health and promoting resilience in this particularly challenging time. Another author (JD) was charged with making a digital video to help new applicants understand what to expect in fellowship at his institution since in person interviews were canceled. Increased virtual didactic sessions and journal clubs were also implemented during the periods of decreased elective surgery.

\section{Unforeseen Opportunities}

The increased use of online didactics and additional time also resulted in additional opportunities for professional and personal development for trainees [33]. We have personally noticed an overall increased time window for literature review and background reading, work on clinical research projects, and manuscript writing time. Furthermore, the pandemic provided new opportunities for clinical research. It is unclear what if any benefits this may have for this generation of surgical trainees, as the balance shifted from the technical to the cognitive aspects of training.

\section{Future Generations of Breast Surgery Fellows}

Effects of this pandemic on breast surgery fellows are likely quite different for different generations of trainees. Recently graduating fellows, such as ourselves, may have experienced a significant impact on case numbers if they were in affected areas in the springtime. Current breast surgery fellows may see similar effects if they are training in the highly affected institutions at present. However, as we learn more about the virus, its spread and its implications, these experiences may 
change, and breast surgery fellowships may be affected at different degrees. This was already seen during the 2020 interview season. The so far biggest impact of the pandemic for future fellows was the change to almost completely remote interviews [34]. This was overall perceived positively and resulted in increased efficiency due to less travel time and decreased overall cost [35]. On average, each candidate saved 6000 USD during this process [36].

\section{Conclusions}

Breast surgery fellowships last for one year and have a strict set of minimum requirements. As a short fellowship based on mostly elective cases, its content was vulnerable to changes in practice patterns associated with the COVID-19 pandemic. The range of effects on breast surgery fellowships was broad and dependent on geographical location, prevalence of institutionalized patients with active COVID-19 infections, and individual institutional policies. Unforeseen practice changes arose with the delay of elective surgical care, increased utilization of telehealth medicine, and the need for social distancing. Inventive academic opportunities such as virtual educational contents, simulation training, increased subspecialty lecture frequency, and additional time to pursue personal and professional developments have contributed positively to the breast fellow's development and may give direction for future approaches to fellowship education. Flexibility, adaptability, and dedication to education have the potential to overcome unforeseen challenges to a training, such as those that occurred during the COVID-19 pandemic.

Acknowledgements The authors would like to thank John Davis MD, Kevin Baratta MD MPH, Noran Barry MD, and Ipshita Prakash MD for their contributions to this article.

\section{References}

Papers of particular interest, published recently, have been highlighted as:

- Of importance

• Of major importance

1. Yan Z. Unprecedented pandemic, unprecedented shift, and unprecedented opportunity. Hum Behav Emerg Technol. 2020 Apr 6: https://doi.org/10.1002/hbe2.192

2. Tanne JH. Covid-19: US cases are greatly underestimated, seroprevalence studies suggest. BMJ. 2020;370:m2988.

3. Luther A, Agrawal A. A practical approach to the management of breast cancer in the COVID-19 era and beyond. Ecancermedicalscience. 2020;14:1059. This article provides an overview of breast cancer management strategies during COVID-19 and discusses implication for future breast cancer care.

4. Society of Surgical Oncology. 2019 Breast Surgical Oncology Fellowship Curriculum and Minimum Training Requirements 2019 [cited 2020 Aug 31]. Available from: https://www.surgo nc.org/wp-content/uploads/2019/06/2019-Breast-Curriculumand-Training-Requirements-Final.pdf.

5.• Hwang ES, Balch CM, Balch GC, Feldman SM, Golshan M, Grobmyer SR, et al. Surgical oncologists and the COVID-19 pandemic: guiding cancer patients effectively through turbulence and change. Ann Surg Oncol. 2020;27(8):2600-13. This paper presents current strategies that have allowed instutions to successfully prepare for oncologic care during the COVID19 pandemic and discusses management of anticipated challenges in the foreseeable future.

6. Garg PK, Kaul P, Choudhary D, Turaga KK, Singh MP, Tiwari AR, et al. Discordance of COVID-19 guidelines for patients with cancer: a systematic review. J Surg Oncol. 2020 Jul 15: https:// doi.org/10.1002/jso.26110

7.• Dietz JR, Moran MS, Isakoff SJ, Kurtzman SH, Willey SC, Burstein HJ, et al. Recommendations for prioritization, treatment, and triage of breast cancer patients during the COVID-19 pandemic. the COVID-19 pandemic breast cancer consortium. Breast Cancer Res Treat. 2020;181(3):487-97. These recommendations from the COVID-19 pandemic breast cancer consortium aim to guide breast cancer care during the current pandemic.

8. Lou N. Sacrifices in Graduate medical education during COVID192020 [cited 202025 Aug]. Available from: https://www. medpagetoday.com/infectiousdisease/covid19/86200.

9. Johns Hopkins University Coronavirus Resource Center. COVID-19 United States cases by county 2020 [cited 2020 Sep 2]. Available from: https://coronavirus.jhu.edu/us-map.

10. Yin K, Singh P, Drohan B, Hughes KS. Breast imaging, breast surgery, and cancer genetics in the age of COVID-19. Cancer. 2020 Oct $15 ; 126(20): 4466-4472$. This study uses data from a risk assessment company to analyze the effect of COVID-19 on the use of breast imaging, breast surgery, and genetic counseling services.

11. Minami CA, Kantor O, Weiss A, Nakhlis F, King TA, Mittendorf EA. Association between time to operation and pathologic stage in ductal carcinoma in situ and early-stage hormone receptor-positive breast cancer. J Am Coll Surg. 2020 Oct;231(4):434-447.

12. Bleicher RJ, Ruth K, Sigurdson ER, Beck JR, Ross E, Wong YN, et al. Time to surgery and breast cancer survival in the United States. JAMA Oncol. 2016;2(3):330-9.

13. Tzeng CD, Teshome M, Katz MHG, Weinberg JS, Lai SY, Antonoff MB, et al. Cancer surgery scheduling during and after the COVID-19 first wave: the MD Anderson Cancer Center experience. Ann Surg. 2020;272(2):e106-11.

14. Aziz H, James T, Remulla D, Sher L, Genyk Y, Sullivan ME, et al. Effect of COVID-19 on surgical training across the United States: a national survey of general surgery residents. J Surg Educ. 2020.

15. Tolu LB, Feyissa GT, Ezeh A, Gudu W. Managing resident workforce and residency training during COVID-19 pandemic: scoping review of adaptive approaches. Adv Med Educ Pract. 2020;11:527-35.

16. Chao TN, Frost AS, Brody RM, Byrnes YM, Cannady SB, Luu $\mathrm{NN}$, et al. Creation of an interactive virtual surgical rotation for undergraduate medical education during the COVID-19 pandemic. J Surg Educ. 2021 Jan-Feb;78(1):346-350.

17. van Dorn A. COVID-19 and readjusting clinical trials. Lancet. 2020;396(10250):523-4. 
18. Kuerer HM, Blair SL. The virtual scientific sessions from the American Society of Breast Surgeons during the COVID-19 pandemic. Ann Surg Oncol. 2020;1-3.

19. Cahan E. Clinical trials rebound after COVID-19 crash, but can enrollment gains continue? 2020 [cited 2020 Sep 20]. Available from: https://www.sciencemag.org/news/2020/07/clinical-trialsrebound-after-covid-19-crash-can-enrollment-gains-continue.

20. Upadhaya S, Yu JX, Oliva C, Hooton M, Hodge J, HubbardLucey VM. Impact of COVID-19 on oncology clinical trials. Nat Rev Drug Discov. 2020;19(6):376-7.

21. Spinner J. Medidata: COVID-19 forcing trials to adapt. [cited 2020 Sep 4]. Available from: https://www.outsourcing-pharma. com/Article/2020/08/04/Medidata-COVID-19-forcing-clini cal-trials-to-adapt.

22. NIH-supported research survey to examine impact of COVID19 on rare diseases community: National Institute of Health; 2020 [cited 2020 Sep 21]. Available from: https://www.nih.gov/ news-events/news-releases/nih-supported-research-survey-exami ne-impact-covid-19-rare-diseases-community.

23. Chen J. Covid-19 has shuttered scientific labs. It could put a generation of researchers at risk 2020 [cited 2020 Sep 21]. Available from: https://www.statnews.com/2020/05/04/coronavirus-labshutdowns-impact-on-scientists-research-delays/.

24. Olayiwola JN, Magaña C, Harmon A, Nair S, Esposito E, Harsh $\mathrm{C}$, et al. Telehealth as a bright spot of the COVID-19 pandemic: recommendations from the virtual frontlines ("Frontweb"). JMIR Public Health Surveill. 2020;6(2):e19045.

25. Nemetz ETA, Urbach DR, Devon KM. The art of surgery: balancing compassionate with virtual care. J Med Internet Res. 2020;22(8):e22417.

26. Rosenbaum L. The Untold Toll - The Pandemic's Effects on Patients without Covid-19. N Engl J Med. 2020;382(24):2368-71.

27. Kilgore L. Breast surgery fellow: why my coronavirus diagnosis led me to donate plasma 2020 [cited 202025 Aug]. Available from: https://www.mdanderson.org/cancerwise/breast-surge ry-fellow-why-my-covid-19-coronavirus-diagnosis-led-me-todonate-convalescent-plasma.h00-159381945.html.
28. Zhang L, Zhu F, Xie L, Wang C, Wang J, Chen R, et al. Clinical characteristics of COVID-19-infected cancer patients: a retrospective case study in three hospitals within Wuhan. China Ann Oncol. 2020;31(7):894-901.

29. Cinar P, Kubal T, Freifeld A, Mishra A, Shulman L, Bachman J, et al. Safety at the time of the COVID-19 pandemic: how to keep our oncology patients and healthcare workers safe. J Natl Compr Canc Netw. 2020;1-6.

30. Qi L, Wang K, Ye C, Zheng S. Special issues encountered when cancer patients confront COVID-19. Front Oncol. 2020;10:1380.

31. Lebares CC, Guvva EV, Ascher NL, O'Sullivan PS, Harris HW, Epel ES. Burnout and stress among US surgery residents: psychological distress and resilience. J Am Coll Surg. 2018;226(1):80-90.

32. Collins C, Mahuron K, Bongiovanni T, Lancaster E, Sosa JA, Wick E. Stress and the surgical resident in the COVID-19 pandemic. J Surg Educ. Mar-Apr 2021;78(2):422-430.

33. de Berker HT, Bressington MJ, Mayo IM, Rose A, Honeyman C. Surgical training during the COVID-19 pandemic: challenges and opportunities for junior trainees. J Plast Reconstr Aesthet Surg. 2021 Mar; 74(3): 644-710.

34. Hill MV, Bleicher RJ, Farma JM. A how-to guide: virtual interviews in the era of social distancing. J Surg Educ. 2021 JanuaryFebruary; 78(1): 321-323

35. Vining CC, Eng OS, Hogg ME, Schuitevoerder D, Silverman RS, Yao KA, et al. Virtual surgical fellowship recruitment during COVID-19 and its implications for resident/fellow recruitment in the future. Ann Surg Oncol. 2020;1-5.

36. Tseng J. How has COVID-19 affected the costs of the surgical fellowship interview process? J Surg Educ. Sep-Oct 2020;77(5):999-1004

Publisher's Note Springer Nature remains neutral with regard to jurisdictional claims in published maps and institutional affiliations. 